\title{
Driving cycle development for Kuala Terengganu city using $k$-means method
}

\author{
I. N. Anida, A.R. Salisa
}

School of Ocean Engineering, Universiti Malaysia Terengganu, Malaysia

\begin{tabular}{l} 
Article Info \\
\hline Article history: \\
Received May 30, 2018 \\
Revised Nov 27, 2018 \\
Accepted Dec 15, 2018
\end{tabular}

\section{Keywords:}

Driving cycle

Fuel rate

Gas emissions

$K$-means

PHEV

\begin{abstract}
Driving cycle plays a vital role in the production and evaluating the performance of the vehicle. Driving cycle is a representative speed-time profile of driving behavior of specific region or city. Many countries has developed their own driving cycle such as United State of America, United Kingdom, India, China, Ireland, Slovenia, Singapore, and many more. The objectives of this paper are to characterize and develop driving cycle of Kuala Terengganu city at 8.00 a.m. along five different routes using $k$-means method, to analyze fuel rate and emissions using the driving cycle developed and to compare the fuel rate and emissions with conventional engine vehicles, parallel plug-in hybrid electric vehicle, series plug-in hybrid electric vehicle and single split-mode plug-in hybrid electric vehicle. The methodology involves three major steps which are route selection, data collection using on-road measurement method and driving cycle development using $k$-means method. Matrix Laboratory software (MATLAB) has been used as the computer program platform in order to produce the best driving cycle and Vehicle System Simulation Tool Development (AUTONOMIE) software has been used to analyze fuel rate and gas emission. Based on the findings, it can be concluded that, Route $\mathrm{C}$ and single spilt-mode PHEV powertrain used and emit least amount of fuel and emissions.
\end{abstract}

Copyright (C) 2019 Institute of Advanced Engineering and Science. All rights reserved.

\section{Corresponding Author:}

A.R. Salisa,

Department of Electrical and Computer Engineering,

School of Ocean Engineering, Universiti Malaysia Terengganu,

21030 Kuala Terengganu, Terengganu, Malaysia.

Email: salisa@umt.edu.my

\section{INTRODUCTION}

The world today is experiencing the alarming level of pollution, especially air pollution made by exhaust emission from the vehicles. Department of Statistics Malaysia has reported that in 2016, motor vehicles have contributed the major sources of air pollution which is $70 \%$. Hybrid vehicle is the most promising vehicle to reduce the fuel consumption and exhaust gas emission [1]. There are a few types of hybrid vehicle such as hybrid electric vehicle (HEV), battery electric vehicle (BEV), full electric vehicle (EV) and plug-in hybrid vehicle (PHEV). Main focus of this paper is PHEV. PHEV is a rechargeable HEV that are equipped with charging connector, typically in-board charger [2]. However, for pioneering vehicle emission models and powertrain input, the development of driving cycle is important [3].

Vehicle driving cycle is a series of point for speed of vehicle versus time which is mainly used to evaluate the performance of either the vehicle or engine. Most of the researches on driving cycle are based on condition of vehicle in a specific location such as United States FTP75, European ECE15, Japan 10115 and others. These driving cycle are widely applied for evaluating the performance of the vehicle emission, fuel consumption, traffic condition and also for designing, development and modeling the new vehicle 
especially hybrid vehicle [4], [5]. However, a driving cycle that is representing Kuala Terengganu city, capital city of Terengganu, has not been established yet.

Three major steps to construct a driving cycle are route selection, data collection and cycle construction. There are various methods of cycle construction. However, four typical and famous methods are micro-trips based construction, pattern classification cycle construction, segment based cycle construction and also modal cycle construction. As in work of [6], [7], the micro-trips based approach has been chosen where the relative error between target and test micro-trips is calculated and the percentage below $15 \%$ is accepted and combined as a driving cycle. Likewise in [8], a semi-random approach is taken on selecting the micro-trips from the road type and timings by classified categories. As in Slovenia, the microtrips are selected from the database and connected in a series to form a driving cycle in such a way that speed-acceleration frequency distribution of the driving cycle is matched to the frequency distribution from the collected speed-time data [9]. While in Dublin, Ireland, a stochastic and statistical methodology is used to develop and assess the representativeness of the driving cycle [10].

For this paper, the micro-trips clustering approach using $k$-means method is used to develop a Kuala Terengganu driving cycle. The differences between this work and others are, the value of $k$ is different regarding the traffic condition and style of driving, and the target route also different. The objectives of this paper are to characterize and develop driving cycle of Kuala Terengganu city at 8.00 a.m. along five different routes using $k$-means method, to analyze fuel economy and emissions using the driving cycle developed and to compare the fuel economy and emissions with conventional engine vehicles, parallel PHEV, series PHEV and split single mode PHEV. In this paper, the development of Kuala Terengganu along five routes will be discussed. In section 2, the methodology including the route selection, data collection, driving cycle development which is by using micro-trips and $k$-means approach also will be discussed. Later, in section 3 , the analysis of the fuel economy and exhaust emissions of PHEV is compared with the analysis of the other vehicles powertrain using Vehicle System Simulation Tool Development (AUTONOMIE) software.

\section{RESEARCH METHOD}

Figure 1 shows the flow chart and research activities on how to develop a driving cycle in Kuala Terengganu (KT) along five different routes which are Route A, Route B, Route C, Route D and Route E during Go-to-Work time in Kuala Terengganu city which is $8.00 \mathrm{am}$. The inputs of KT driving cycle are second-by-second speed. The data are collected at Go-to-Work (GTW) time which is 8.00 am since 8.00 am is the mostly people in Kuala Terengganu go to work with 10 runs of data. All five routes have been chosen as selected routes based on its traffic volume in Kuala Terengganu. In this research, the on-board measurement method will be used using a Global Positioning System (GPS). The data gathered then will be divided into several micro-trips and from each micro-trip, the features such as average speed and percentage of idle will be calculated. The clustering of the micro-trips using $k$-means method will be took place in order to find the final driving cycle of Kuala Terengganu along Route A, B, C, D and E at 8.00 a.m. Then, using the final driving cycle of Kuala Terengganu, the fuel economy and emissions will be analyzed using AUTONOMIE software and will be compared to other vehicles' powertrain such as conventional engine vehicle, parallel PHEV, series PHEV and split single mode PHEV.

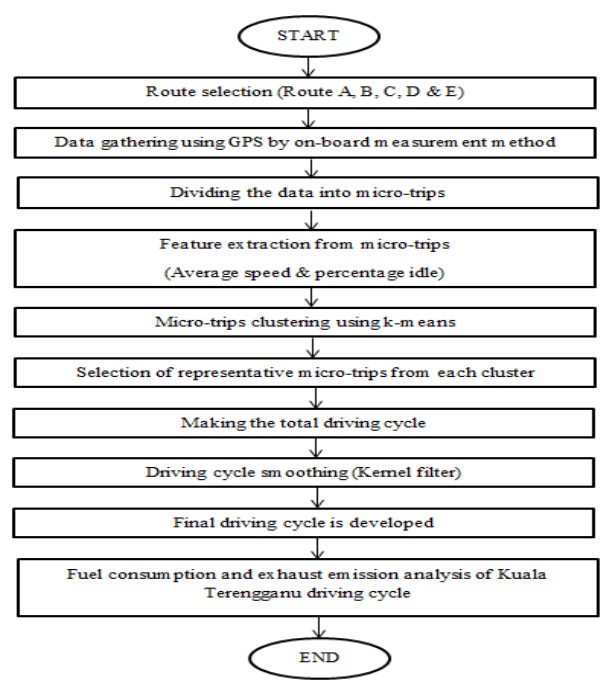

Figure 1. Flow chart of driving cycle development 
Table 1 shows the assessment parameters in order to characterize the driving data. The parameters chosen are average speed, average driving speed, average acceleration and deceleration, time proportion of idling, cruising, acceleration and deceleration and root mean square of acceleration. Those 9 parameters have been chosen as the assessment parameters since they are the fundamental assessment in order to determine the characterization of the driving cycle.

Table 1. KT Driving Cycle Assessment Parameters

\begin{tabular}{|c|c|c|}
\hline Parameters & Unit & Equation \\
\hline Average speed of whole driving cycle & $\mathrm{Km} / \mathrm{h}$ & $\mathrm{V}_{1}=3.6 \frac{\mathrm{dist}}{\mathrm{T}_{\text {total }}}$ \\
\hline Average running speed & $\mathrm{Km} / \mathrm{h}$ & $\mathrm{V}_{2}=3.6 \frac{{ }_{\mathrm{T}}^{\text {dist }}}{\mathrm{T}_{\mathrm{drive}}}$ \\
\hline Average acceleration of all acceleration phase & $\mathrm{m} / \mathrm{s}^{2}$ & $\begin{array}{l}\mathrm{a}=\left(\sum_{i=1}^{n}\left\{\begin{array}{cc}1 & \left(a_{i}>0\right) \\
0 & (\text { else })\end{array}\right)\right)^{-1} \\
\left(\sum_{i=1}^{n}\left\{\begin{array}{cc}a_{i}\left(a_{i}>0\right) \\
0 & (\text { else })\end{array}\right)\right.\end{array}$ \\
\hline Average deceleration of all deceleration phase & $\mathrm{m} / \mathrm{s}^{2}$ & $\begin{array}{l}\mathrm{d}=\left(\sum_{i=1}^{n}\left(\begin{array}{cc}1 & \left(a_{i}<0\right) \\
0 & (\text { else })\end{array}\right)^{-1}\right. \\
\left(\sum_{i=1}^{n}\left\{\begin{array}{c}a_{i}\left(a_{i}<0\right) \\
0(\text { else })\end{array}\right)\right.\end{array}$ \\
\hline Time proportion of idling & $\%$ & $\%$ idle $=\frac{T_{\text {idle }}}{T_{\text {tota }}}$ \\
\hline Time proportion of cruising & $\%$ & $\%$ cruise $=\frac{T_{\text {cruise }}}{T_{\text {ton }}}$ \\
\hline Time proportion of acceleration & $\%$ & $\%$ acc $=\frac{T_{\text {acc }}}{T_{\text {total }}}$ \\
\hline Time proportion of deceleration & $\%$ & $\% \operatorname{dec}=\frac{\frac{T_{\text {Tdec }}}{T_{\text {total }}}}{2}$ \\
\hline Root mean square acceleration & $\mathrm{m} / \mathrm{s}^{2}$ & $\mathrm{RMS}=\sqrt{\frac{1}{T} \int_{0}^{T}(a)^{2} d t}$ \\
\hline
\end{tabular}

\subsection{Route selection}

Figure 2 highlights the selected routes for KT driving cycle from Kampung Wakaf Tembesu to Wisma Persekutuan named as Route A, Route B, Route C, Route D and Route E. According to Ministry of Works Malaysia, these five routes are the most frequent routes used by Kuala Terengganu citizen as 'routeto-work' routes [11]. In this study, speed-time data are collected by using GPS based on on-board measurement method along the selected route starting from Kampung Wakaf Tembesu to Wisma Persekutuan. Kampung Wakaf Tembesu as the starting point is chosen due to its population. Whereas, Wisma Persekutuan as the end point is chosen because most of the government sectors located in the Wisma Persekutuan and it's nearby.

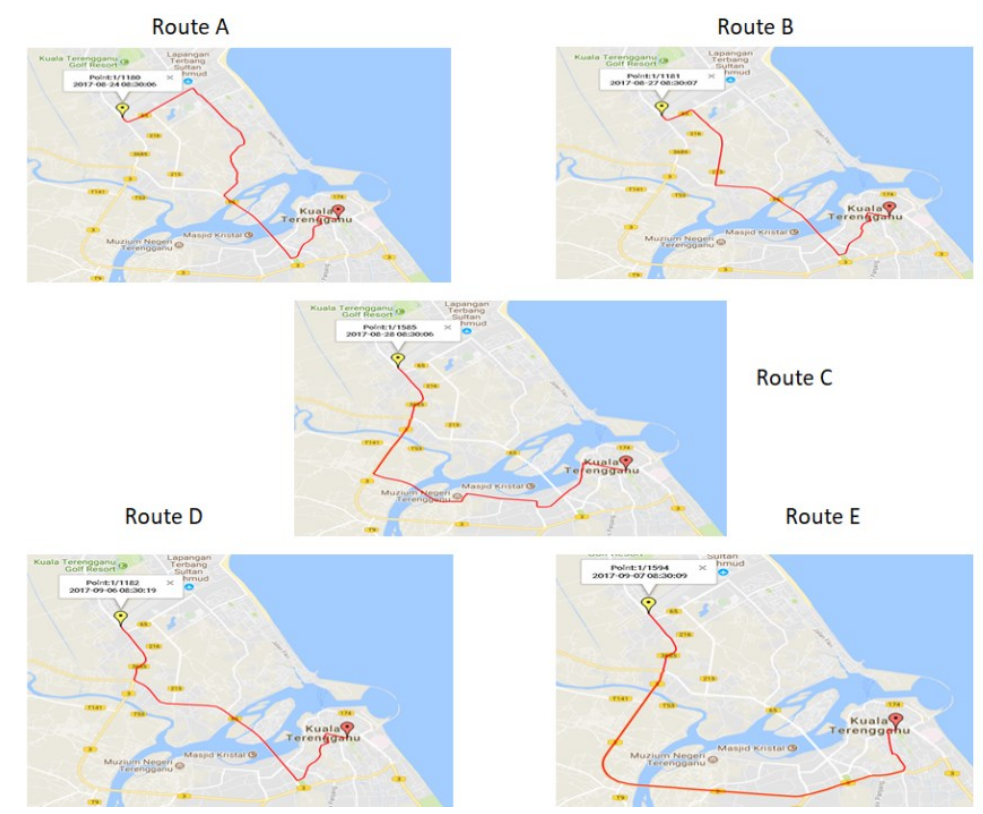

Figure 2. Selected routes; Route A, B, C, D and E 


\subsection{Data collection and micro-trips definition}

Data was collected at GTW time which is 8.00 am along the selected road with 10 runs. There are three types of techniques or ways to collect the data which are chase car technique, on-board measurement technique and combination of on-board measurement and circulation driving. Chase car technique is when instrumented vehicle record the second-by-second speed data as it follows the target vehicles. While onboard measurement technique is when speed-time data collections were carried out using a real time logging system equipped on a selected vehicle along the predetermined route. Lastly, combination of on-board measurement and circulation driving also known as hybrid method is the combination of the two techniques [12]. For KT driving cycle, on-board measurement technique will be used for the data collection since it is more suitable for KT drivers' irregular behavior to avoid a risk such as accident and sudden loss of control.

The development of a drive cycle is based on micro trips. Micro-trip is a trip between two successive time points at which the vehicle velocity is zero [13]. Each micro trip starts with an idle phase and ends with a decelerating phase which reduces to zero. This measure of motion involves of acceleration, cruise and deceleration modes. The whole data has to be separated into number of micro-trips. A large number of micro trips can be acquired after this process for all collected data. Then, the micro-trips are clustered into several groups depending on the traffic situations such as congested traffic flow, medium traffic flow and clear traffic flow. $K$-means approach will be using in order to cluster the micro-trips.

\section{3. $K$-means clustering method}

As mentioned earlier, in this study, $k$-means approach will be used in order to cluster the micro-trips since it is simple and fast method yet easy to implement. $K$-means is one of the simplest unsupervised learning algorithms that solve the clustering problem. The procedure follows a simple and easy way to classify a given data set through a certain number of clusters (assume $k$ clusters) fixed a priori. The steps of the $k$-means algorithms are described briefly below [14]:

Step 1: Decide on a value for $k$. In this study, the value of $k$ is based on traffic condition.

Step 2: Initialize the $k$ cluster centers (randomly, if necessary)

Step 3: Decide the class memberships of the total data, $\mathrm{N}$ by assigning them to the nearest cluster center.

Step 4: Re-estimate the k-cluster centers, by assuming the memberships found above are correct.

Step 5: If none of the $\mathrm{N}$ data changed memberships in the last iteration, exit. Otherwise go to Step 3.

\subsection{Feature extraction and micro-trips clustering}

The proposed approach in developing the driving cycle is by micro-trips clustering. In order to cluster the micro-trips, driving features must be extracted first. There are a lot of driving features that can be extracted from the micro-trips as mentioned earlier in Table 1. But, for this purpose, only two features will be used which are average speed and also percentage of idle. These two features have been chosen since they will give greatest effect on the emission [15]. After the extraction of the parameters, the average speed and percentage of idle is plotted in 2-dimensional feature space as in Figure 3. As shown in Figure 3, it can be proved that there is a relation between average speed and percentage of idle. When the average speed is high, the percentage of idle will be low and vice versa.

Figure 4 shows the micro-trips are clustered into three groups using $k$-means clustering method. Each group has its own characteristics and stands for different traffic condition; clear traffic condition, medium traffic condition and congested traffic condition.

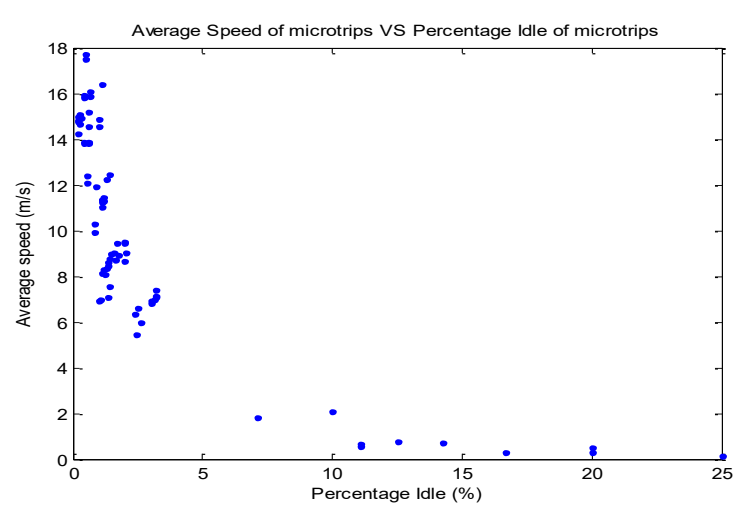

Figure 3. Average speed of micro-trips VS Percentage idle of micro-trips

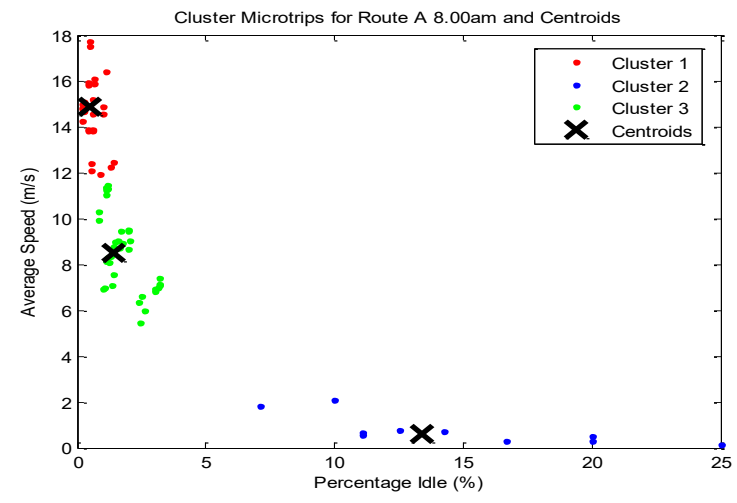

Figure 4. Clustering of micro-trips 


\subsection{KT driving cycle development}

In previous section, the micro-trips have been clustered into 3 groups. Whereas, in this section, the representatives of micro-trips are determined in order to produce the driving cycle for each cluster. The closest micro-trips to the cluster center will consider as the representative micro-trips. The selected micro-trips for each group are presented as in Figure 5, Figure 6 and Figure 7. The micro-trips then will be combined in order to produce final driving cycle of Kuala Terengganu along Route A, B, C, D and E at 8.00 a.m.

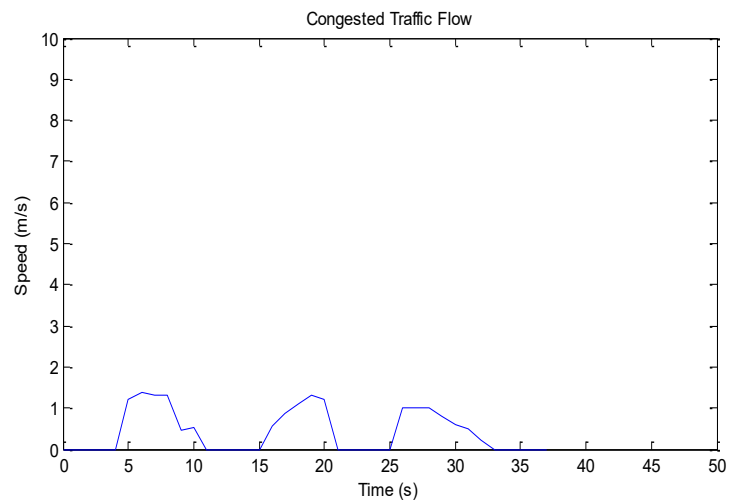

Figure 5. Congested traffic condition

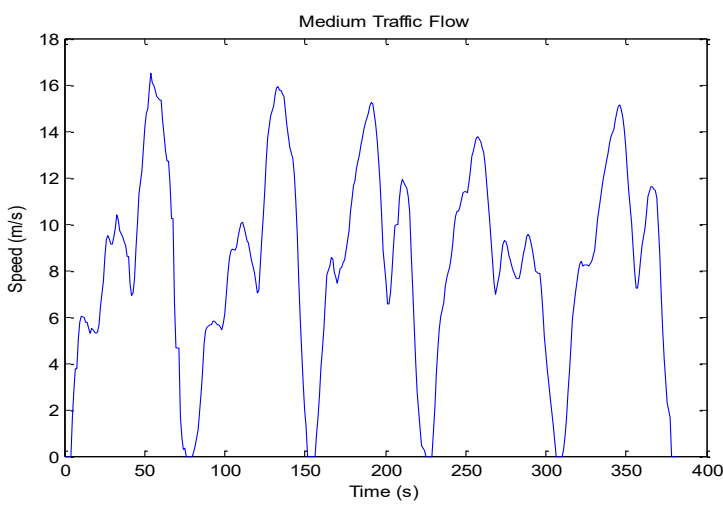

Figure 6. Medium traffic condition

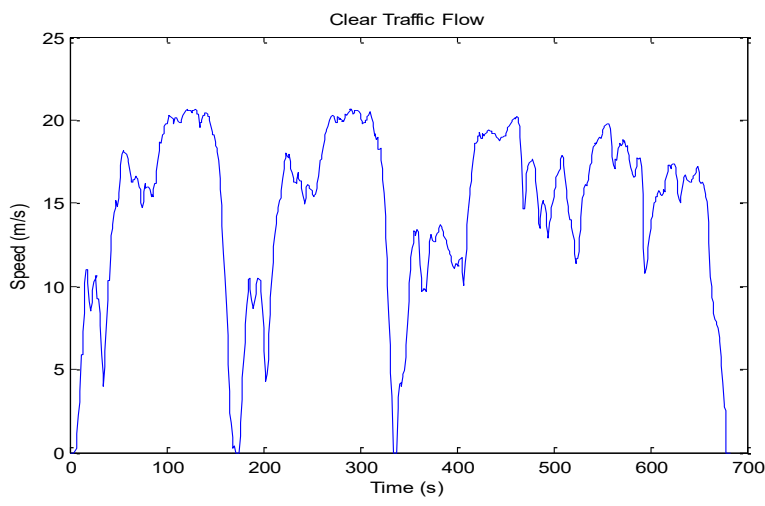

Figure 7. Clear traffic condition

\section{RESULTS AND ANALYSIS}

In this section, the results of the development of driving cycle will be analyzed and discussed. Also, the analysis of the fuel rate and gas emission will be determined and the comparison between KT driving cycle with other vehicle powertrain such as conventional engine vehicle, parallel PHEV, series PHEV and split single mode PHEV will also be discussed.

\subsection{KT driving cycle analysis}

Figures 8-12 shows the final proposed Kuala Terengganu driving cycle along Route A, B, C, D, and $\mathrm{E}$ at 8.00 a.m. As in the figure, it shows that every routes present different pattern of driving cycle. This is due to other external factors such as traffic light, road conditions, drivers' behavior and environmental factors [16]. Also, the micro-trips at the higher speed range are longer compared to the micro-trips at lower speed range. This is because the vehicle experiencing a free flow moves at a higher speed range with less frequent stop due to less traffic condition. 


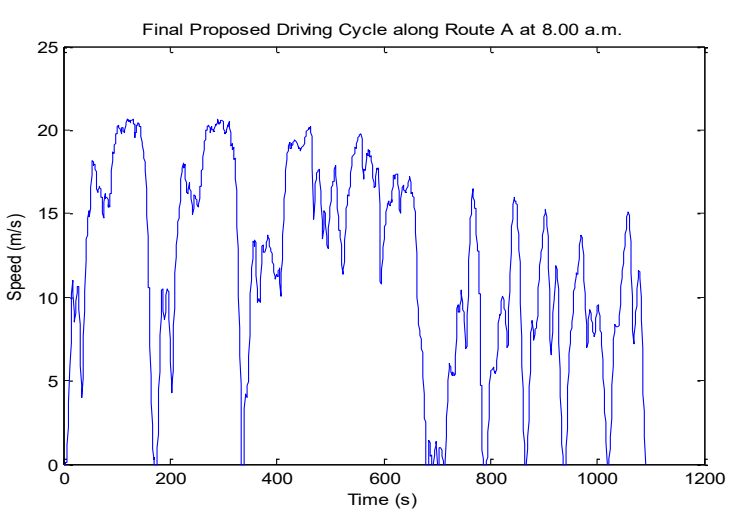

Figure 8. Final proposed of KT driving cycle along Route A

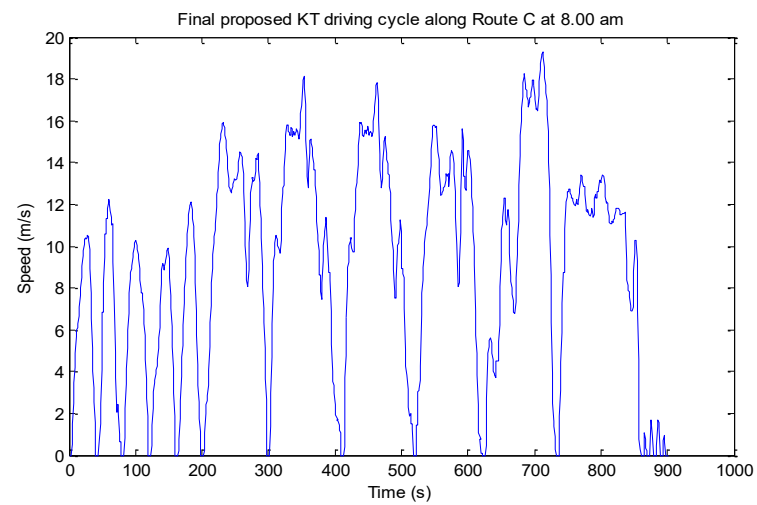

Figure 10. Final proposed of KT driving cycle along Route C

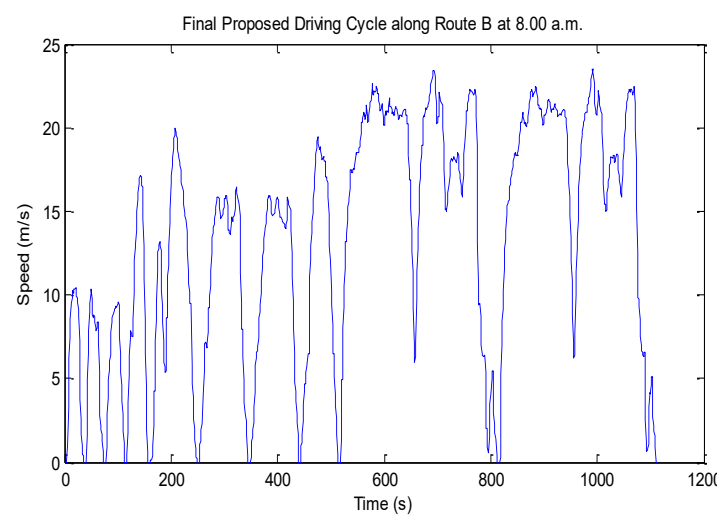

Figure 9. Final proposed of KT driving cycle along Route B

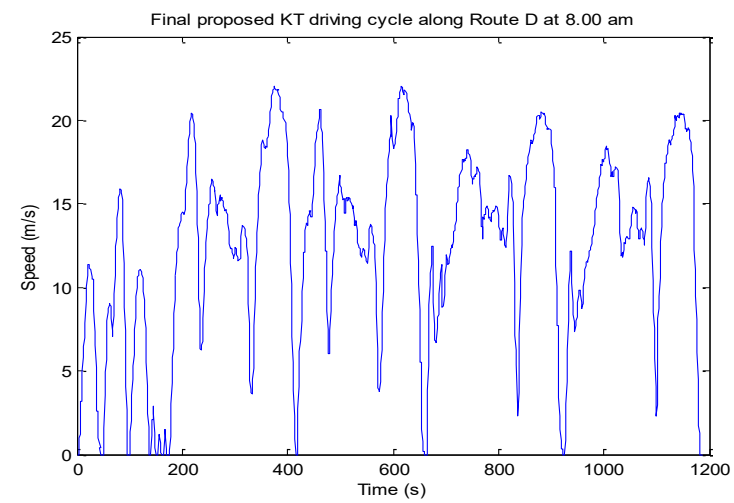

Figure 11. Final proposed of KT driving cycle along Route D

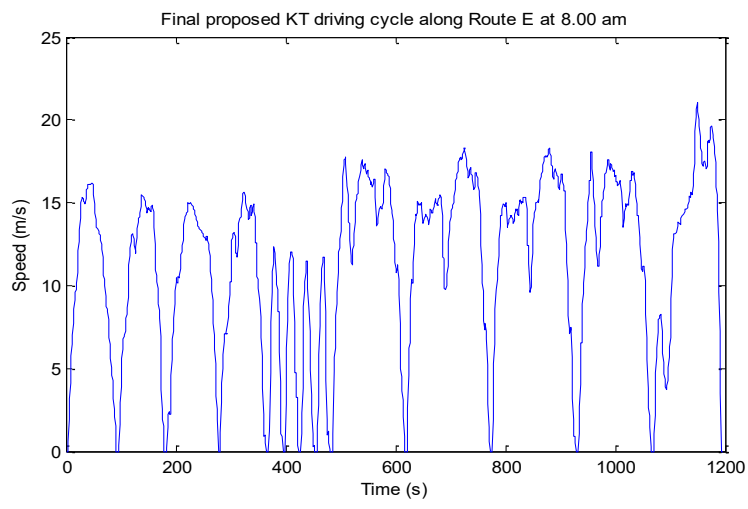

Figure 12. Final proposed of KT driving cycle along Route E

Table 2 shows the characteristics of KT driving cycle along Route A, B, C, D and E in terms of nine parameters such as average speed, average driving speed, average acceleration and deceleration, time proportion of idling, cruising, acceleration and deceleration and root mean square of acceleration. From the table, it shows that the speed range above $30 \mathrm{~km} / \mathrm{h}$ was dominant. This is due to the busy and medium traffic condition of KT 'route-to-work' routes. 
Table 2. Assessment Parameters of KT Driving Cycle along Route A, B, C, D and E

\begin{tabular}{llllll}
\hline Parameters & Route A & Route B & Route C & Route D & Route E \\
\hline Distance travelled $(\mathrm{km})$ & 13.11 & 14.85 & 8.21 & 14.94 & 13.61 \\
Total time $(\mathrm{s})$ & 1094 & 1114 & 901 & 1187 & 1197 \\
Average speed $(\mathrm{km} / \mathrm{h})$ & 43.13 & 47.99 & 32.80 & 45.29 & 40.93 \\
Average running speed $(\mathrm{km} / \mathrm{h})$ & 45.63 & 50.48 & 36.00 & 47.49 & 43.47 \\
Average acceleration $\left(\mathrm{m} / \mathrm{s}^{2}\right)$ & 0.44 & 0.49 & 0.55 & 0.48 & 0.49 \\
Average deceleration $\left(\mathrm{m} / \mathrm{s}^{2}\right)$ & 0.51 & 0.57 & 0.57 & 0.54 & 0.49 \\
RMS $\left(\mathrm{m} / \mathrm{s}^{2}\right)$ & 0.64 & 0.72 & 0.71 & 0.70 & 0.68 \\
Percentage idle $(\%)$ & 4.47 & 4.04 & 7.21 & 3.79 & 4.76 \\
Percentage cruise $(\%)$ & 2.92 & 7.17 & 5.43 & 7.49 & 5.59 \\
Percentage acceleration $(\%)$ & 49.59 & 47.80 & 44.46 & 47.05 & 44.49 \\
Percentage deceleration $(\%)$ & 43.01 & 40.99 & 42.90 & 41.67 & 45.16 \\
\hline
\end{tabular}

\subsection{Fuel rate and emissions analysis}

After the driving cycle has been developed, the fuel rate such as fuel consumption and fuel economy, and emission can be determined using AUTONOMIE software version v1210. AUTONOMIE is a tool for automotive control system design, simulation and analysis. It is mathematically-based forward simulation software based on MATLAB, with MATLAB data and configuration files and models built in Simulink. Table 3 shows the fuel rate and emissions of KT driving cycle for Route A, B, C, D, and E using split single mode PHEV powertrain. The emission of a vehicle will emit carbon monoxide $\left(\mathrm{CO}_{2}\right)$ gas.

From the result in the table, it can be concluded that Route $\mathrm{C}$ is the best route to take in order to go to Wisma Persekutuan at 8.00 a.m. since it gives the lowest value of fuel consumption which is 0.49 $1 / 100 \mathrm{~km}$, the highest value of fuel economy which is $202.14 \mathrm{~km} / 1$ and the lowest value of $\mathrm{CO}_{2}$ emission which is $11.68 \mathrm{~g} / \mathrm{km}$. While, Route B gives the highest value of fuel consumption and emission which are $1.35 \mathrm{l} / 100 \mathrm{~km}$ and $31.96 \mathrm{~g} / \mathrm{km}$ respectively, with the lowest value of fuel economy which $73.90 \mathrm{~km} / \mathrm{l}$.

Table 3. Fuel Rate and Emissions of KT Driving Cycle

\begin{tabular}{cccc}
\hline Route & Fuel consumption $(1 / 100 \mathrm{~km})$ & Fuel economy $(\mathrm{km} / \mathrm{l})$ & $\mathrm{CO}_{2}$ Emission $(\mathrm{g} / \mathrm{km})$ \\
\hline $\mathrm{A}$ & 0.55 & 181.32 & 13.03 \\
$\mathrm{~B}$ & 1.35 & 73.90 & 31.96 \\
$\mathrm{C}$ & 0.49 & 202.14 & 11.68 \\
$\mathrm{D}$ & 0.92 & 108.94 & 21.68 \\
$\mathrm{E}$ & 0.54 & 184.36 & 12.81 \\
\hline
\end{tabular}

In order to prove that split single mode of PHEV gives the lowest value of fuel consumption and $\mathrm{CO}_{2}$ emission, and the highest value of fuel economy, the comparison between conventional engine vehicles, parallel PHEV, series PHEV and split single mode PHEV has been made using Route C driving cycle and tabulated in Table 4. From the table, it clearly shows that split single mode of PHEV is the best powertrain among conventional engine, parallel PHEV and series PHEV with the lowest value of fuel consumption and emission, and the highest value of fuel economy. It is because, the power split type PHEV is designed by combining one engine, two motor-generators (MGs), and multiple planetary gears [17]. Thus, it will reduce the emissions emitted and the energy consumption.

Table 4. Comparison between Split Single Mode PHEV, Parallel PHEV, Series PHEV and Conventional Vehicle

\begin{tabular}{lcccc}
\hline & Split single mode PHEV & Parallel PHEV & Series PHEV & Conventional vehicle \\
\hline Fuel Economy $(\mathrm{km} / \mathrm{l})$ & 202.14 & 102.63 & 63.24 & 14.02 \\
Fuel Consumption $(1 / 100 \mathrm{~km})$ & 0.49 & 0.97 & 1.58 & 7.13 \\
$\mathrm{CO} 2$ Emission $(\mathrm{g} / \mathrm{km})$ & 11.68 & 23.05 & 37.41 & 168.79 \\
\hline
\end{tabular}

\section{CONCLUSION}

The development of KT driving cycle is done using micro-trips clustering by $k$-means method. The data are collected from predetermined initial location to final location along Route A, B, C, D and E at Goto-Work time which is $8.00 \mathrm{am}$. The KT driving cycle is successfully obtained and can be concluded that the proposed method is possible to generate a KT driving cycle for PHEV powertrain to overcome exhaust emission and fuel economy problems. Further study has to be made on other main road in Kuala Terengganu city. 


\section{ACKNOWLEDGEMENTS}

All the gratitude and acknowledgement goes to the financial support of this work by the Fundamental Research Grant Scheme vot 59453 and the Universiti Malaysia Terengganu

\section{REFERENCES}

[1] N. Murgovski, L. Johannesson, J. Sjoberg, and B. Egardt, "Component sizing of a plug-in hybrid electric powertrain via convex optimization," Mechatronics, vol. 22, pp. 106-120, 2012.

[2] S. Ou, Y. Zhou, J. Lian, P. Jia, and B. Tian, "Development of Hybrid City Bus 's Driving Cycle," 2011 International Conference on Electric Information and Control Engineering (ICEICE) , pp. 1-5, 2011.

[3] I. N. Anida, A. Z. Fathonah, W.H. Atiq, J. S. Norbakyah, and A. R. Salisa, "Driving cycle analysis for fuel economy and emissions in Kuala Terengganu during peak time," Journal of Telecommunication, Electric and Computer Engineering, vol. 10, pp. 2-5, 2018.

[4] Shi, Q. et al., "The study of a new method of driving cycles construction," International Workshop on Automobile, Power and Energy Engineering, vol. 16, pp. 79-87, 2011.

[5] V. Schwarzer and R. Ghorbani, "Drive Cycle Generation for Design Optimization of Electric Vehicles," IEEE Transactions On Vehicular Technology, vol. 62(1), pp. 89-97, 2013.

[6] N.H. Arun, S. Mahesh, G. Ramadurai, and S.M. Shiva, "Development of driving cycles for passenger cars and motorcycles in Chennai, India," Sustainable Cities and Society, 2017.

[7] G. Amirjamshidi and M.J. Roonda, "Development of simulated driving cycles: case study of the Toronto Waterfront Area," Transportation Research Part D, vol. 34, pp. 255-266, 2015.

[8] S-H. Ho, Y-D. Wong, and V.W-C. Chang, "Developing Singapore driving cycle for passenger cars to estimate fuel consumption and vehicular emissions," Atmospheric Environment, vol. 97, pp. 353-362, 2014.

[9] P. Lipar, I. Strnad, M. Cesnik, and T. Maher, "Development of urban driving cycle with GPS data post processing," Traffic \& Transportation, vol. 28(4), pp. 353-364, 2016.

[10] J. Brady and M. O’Mahony, "Development of a driving cycle to evaluate the energy economy of electric vehicles in urban areas," Applied Energy, vol. 177, pp. 165-178, 2016.

[11] Ministry of Works Malaysia, "2014 Road Traffic Volume Malaysia (RTVM)," Ministry of Works Malaysia, Highway Planning Division, 2015.

[12] U. Galgamuwa, L. Perera, and S. Bandara, "Developing a General Methodology for Driving Cycle Construction Comparison of Various Established Driving Cycles in the World to Propose a General Approach," Journal of Transportation Technologies, vol. 5, pp. 191-203, 2015.

[13] Q. Wang, H. Huo, He. Yao, and Z. Zhang, "Characterization of Vehicle Driving Patterns and Development of Driving Cycles in Chinese Cities," Transportation Research Part D, vol. 13, pp. 289-297, 2008.

[14] U. Maulik and S. Bandyopadhyay, "Genetic algorithm based clustering technique," Pattern Recognition, vol. 33(9), pp. 1455-1465, 2000.

[15] A. Fotouhi and M. Montazeri-Gh, "Tehran driving cycle development using the $k$-means clustering method," Scientia Iranica, Transactions A: Civil Engineering, vol. 20(2), pp. 286-293, 2013.

[16] Andreas Braun and Wolfgang Rid, "The influence of driving patterns on energy consumption in electric car driving and the role of regenerative braking," Transportation Research Procedia, vol. 22, pp. 174-182, 2017.

[17] Hanho Son, Kyusik Park, Sungho Hwang and Hyunsoo Kim, "Design methodology of a power split type plug-in hybrid electric vehicle considering drivetrain losses," Energies 2017, vol. 10, pp. 437, 2017.

\section{BIOGRAPHIES OF AUTHORS}

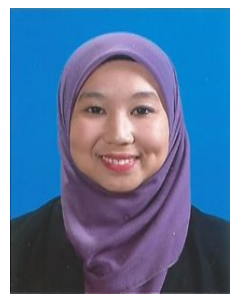

I.N. Anida received the Bachelor of Engineering (Mechatronics) from International Islamic University Malaysia, Gombak in 2015 and currently pursuit her Master Degree in Physics (Renewable Energy) in Universiti Malaysia Terengganu. Her main research interest is Plug in Hybrid Vehicle, driving cycle, energy rate and emissions.

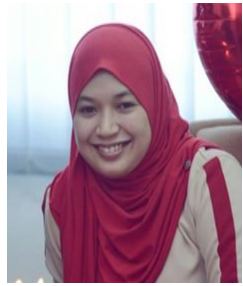

A.R. Salisa received the B.E. and M.E. in Electrical \& Electronics Engineering from University of Technology Petronas, Perak, Malaysia in 2004 and 2006, respectively while Ph.D. in Optimal Energy Management Strategy for the University of Technology Sydney Plug-In Hybrid Electric Vehicles from University of Technology Sydney (UTS), Australia. She is currently working as a senior lecturer at University Malaysia Terengganu, Malaysia. Her research interests are in Hybrid Electric Vehicles, innovation powertrain, simulation and modeling, energy management strategy, driving cycles, fuel economy, emissions and optimization. 